Milena Povolo, Lucia Monti, Valeria Pelizzola, Giovanna Contarini

\title{
Studio sulla composizione del latte d'asina
}

\author{
Study on the donkey millk composition
}

CREA - Centro di Ricerca Zootecnia e Acquacoltura, Via Antonio Lombardo 11, 26900, Lodi, Italy

\section{${ }^{*}$ Corresponding author: \\ Milena Povolo}

CREA-ZA, Via Lombardo 11, 26900 Lodi.

Tel. 0371450163 E-mail: milena.povolo@crea.gov.it
Ricevuto il 20 dicembre 2019

Accettato il 3 febbraio 2020

DOI: $10.36138 /$ STLC.01.2020.03

\section{Riassunto}

Obiettivi: La composizione chimica del latte d'asina è stata studiata da diversi autori, che hanno rilevato contenuti molto variabili dei singoli costituenti. Tali risultati sono spesso ascrivibili al fatto che le analisi sono state effettuate sul latte raccolto, a volte, da singoli animali, di razze diverse, alimentate in modo molto diversificato e con tecnologie di allevamento e mungitura differenti a seconda dell'azienda. Scopo di questo studio è stata la valutazione delle caratteristiche compositive del latte d'asina utilizzando latte massale di un grande allevamento italiano, che direttamente lo commercializza, sia liquido pastorizzato che liofilizzato. Materiali e Metodi: Sul latte di 11 razze diverse sono stati determinati i macrocostituenti, le diverse frazioni proteiche, la composizione in trigliceridi, acidi grassi e fosfolipidi.

Risultati: Tra i principali costituenti, la materia grassa si è confermata l'indice più variabile del latte d'asina (0.2$0.8 \%$ ) ed il meno concentrato tra i macrocomponenti. Tale frazione è pe- rò risultata ricca di composti ad elevato significato dietetico/nutrizionale, quali acidi grassi insaturi della serie omega 3 e fosfolipidi, e priva di acidi grassi in configurazione trans. L'analisi della componente proteica ha confermato il rapporto pressoché unitario tra caseina e sieroproteine, nonché l'elevata concentrazione di lisozima (0.14 g/100ml).

Conclusioni: I dati ottenuti dal lavoro svolto possono essere utili ai medici e nutrizionisti che consigliano questo prodotto in presenza di particolari patologie, non solo in ambito pediatrico. Inoltre, possono contribuire a fare chiarezza e a selezionare le numerose informazioni, spesso di incerta provenienza, che si trovano sul web, in merito al latte d'asina.

\section{Parole chiave: \\ - latte d'asina \\ composizione \\ frazione proteica \\ frazione lipidica \\ valore nutrizionale}

\section{Abstract}

Objective: The chemical composition of donkey milk has been studied by many authors, who detected a high variability of the constituents. These findings can be ascribed to different reasons: the collection of single animal milk, sometimes, the different breed and type of feeding, and the different farm management. The scope of this study was the evaluation of the composition of bulk donkey milk produced, and directly commercialized by a big Italian farm as both pasteurized liquid milk and lyophilized powder.

Materials and methods: The following evaluations were performed on milk samples of 11 different donkey breeds: main components, protein fractions, triglycerides, fatty acids and phospholipids. Results: Among the main components of donkey milk, milk fat demonstrated to be the most variable parameter (0.2-0.8\%) and the less concentrated one. However, this fraction in- cluded a high concentration of compounds having a nutritional/ dietetic significance, i.e. omega 3 fatty acids and phospholipids. No trans fatty acids were found in donkey milk fat. The analysis of proteins demonstrated that, unlike cow milk, the ratio casein/whey proteins was about 1 and the lysozyme was highly concentrated $(0.14 \mathrm{~g} / 100 \mathrm{ml})$. Conclusions: The data obtained by this work can be helpful to doctors and nutritionists who recommend this product in presence of particular diseases, not only in the pediatric field. In addition, this paper can be a source of data able to clarify and select the large number of web information, often of uncertain origin, about donkey millk.
Keywords:
donkey milk
composition
protein fraction
- lipid fraction
nutritional value 


\section{INTRODUZIONE}

II latte d'asina è un alimento funzionale le cui qualità nutrizionali e terapeutiche sono note fin dall'antichità. Tale prodotto viene ormai considerato un ottimo alimento per molte categorie di consumatori in considerazione dell'elevata digeribilità, del suo contenuto in vitamine, sali minerali e zuccheri. Tuttavia, la caratteristica che desta maggiore interesse è la sua composizione chimica molto simile a quella del latte umano (1-3).

Infatti, il contenuto proteico totale del latte d'asina è inferiore (1.5$1.8 \%$ ) rispetto al latte vaccino (3.1-3.8\%) e più simile al latte materno (0.9-1.7\%). In particolare, le proteine del siero di latte rappresentano il $35-50 \%$ della frazione azotata (20\% nel latte vaccino) e quindi il rapporto tra caseina e sieroproteine è inferiore a quello del latte vaccino (1.2 vs 4.7). Anche il contenuto di lattosio (5.8-7.4\%) è molto simile a quello del latte umano (6.3-7.0\%) e molto più elevato rispetto al latte vaccino (4.4-4.9\%) (4). Per contro, il latte d'asina è caratterizzato da una quantità di grasso inferiore $(0,3-1,8 \%)$ rispetto al latte materno (3.5-4.0\%) e il diametro della maggior parte dei globuli di grasso (circa $10 \mu \mathrm{m}$ ) è superiore a quello del latte bovino e umano (rispettivamente 3-3.5 $\mu \mathrm{m}$ e $4 \mu \mathrm{m}$ ) (1).

Questa somiglianza compositiva con il latte materno sembra sia la spiegazione alle proprietà ipoallergeniche e terapeutiche che da sempre sono attribuite al latte d'asina. Negli ultimi anni, è stato dimostrato che questo latte è ben tollerato dai bambini che soffrono di allergia alle proteine del latte vaccino e che può essere un valido sostituto del latte materno qualora questo non sia più disponibile (5). Inoltre, il suo uso potrebbe essere di grande interesse per la prevenzione delle malattie cardiovascolari, nel trattamento delle malattie immunitarie umane e nelle diete ipocolesterolemiche. II contenuto ipolipidico e il corrispondente valore energetico ridotto devono essere presi in considerazione nel bilanciamento nutrizionale, soprattutto se il latte d'asina viene utilizzato nell'alimentazione infantile. Per questo motivo, quando utilizzato come sostituto del latte umano, è di solito integrato con olio vegetale, per compensare l'assunzione di energia. Alcuni studi sulle caratteristiche nutrizionali del latte d'asina, il suo possibile impatto sulla salute umana e le sue potenziali applicazioni nel settore lattiero sono stati recentemente sintetizzati da Carminati e Tidona (6). II sempre crescente interesse verso le caratteristiche funzionali del latte d'asina ha portato, negli ultimi anni, ad un incremento del numero di ricerche sulle caratteristiche compositive di questo prodotto. II ridotto numero di allevamenti, spesso con pochi capi di bestiame e la presenza di numerose razze ha determinato una notevole variabilità dei risultati. Per questo motivo, questa ricerca ha preferito effettuare le valutazioni sul latte massale di uno dei più grandi allevamenti di asine che produce e distribuisce sia latte liquido che liofilizzato. L'obiettivo era fornire un quadro complessivo di una composizione media che tenesse conto sia delle diverse razze, sia delle diverse stagioni. Inoltre, accanto alla determinazione dei parametri compositivi classici, sono state effettuate alcune determinazioni più specifiche finora poco o nulla applicate a questo prodotto.

\section{MATERIALI E METODI}

Campioni di latte d'asina di massa sono stati reperiti, nell'arco di 1 anno, nella principale azienda in Europa specializzata nella produzione di latte di asina, situata nella provincia di Reggio Emilia. II latte derivava da 11 razze diverse, tra le quali le razze Romagnolo e Martina Franca erano le più rappresentate. Dopo la mungitura, i campioni sono stati immediatamente raffreddati, trasportati in laboratorio in condizioni refrigerate, e conservati a $-20^{\circ} \mathrm{C}$ fino al momento dell'analisi. La valutazione del $\mathrm{pH}$ è stata effettuata immediatamente all'arrivo in laboratorio, prima del congelamento. Su ciascun campione sono stati determinati: sostanza secca (7), ceneri (8), proteina totale (9), caseina, azoto non proteico e sieroproteine (10), sostanza grassa (11), zuccheri e acidi organici (12).

L'analisi quali-quantitativa della componente proteica del latte d'asina è stata eseguita in elettroforesi capillare (CE), utilizzando uno strumento P/ACE MDQ Glycoprotein System (Beckman Coulter, Inc., Fullerton, CA). È stata effettuata sia una CE in presenza di Sodio Dodecil Solfato (CE-SDS) (ProteomeLab SDS-MW Analysis kit, Beckman Coulter), sia una CE zonale in presenza di urea (CZEUREA) (13).

Sulla materia grassa estratta utilizzando il metodo di Folch et al. (14) è stata determinata la composizione in trigliceridi (15), acidi grassi (16), fosfolipidi (17), ed è stato valutato il contenuto in colesterolo (18).

\section{RISULTATI E DISCUSSIONE}

La tabella I riporta i dati relativi ai parametri determinati, espressi come valore medio, deviazione standard e coefficiente di variazione. II pH del latte d'asina, a differenza di quello del latte vaccino (6.6-6.8) è neutro o leggermente alcalino, principalmente come conseguenza del basso contenuto di caseine e fosfati. Sono infatti le funzioni acide di questi costituenti che, avendo una costante di dissociazione nell'intervallo della neutralità, si trovano in condizione di equilibrio tra la forma dissociata e indissociata e possono quindi cedere idrogenioni. I valori ottenuti sono in linea con i dati bibliografici riportati da Salimei (2) e Malissiova et al. (19). Gli intervalli di variabilità riportati da questi ultimi autori, sono decisamente più ampi di quelli proposti sia da Salimei (2), Salimei e Fantuz (1) e Carminati e Tidona (6), poiché 
derivano da analisi effettuate su latte di singoli animali. II contenuto in materia grassa, come era ipotizzabile, è risultato il parametro più variabile, con un valore medio $(0.46 \%)$ che si posiziona sui limiti inferiori del range osservato dagli altri autori. Più costante invece il valore delle proteine totali, che si posiziona ben al di sotto dei valori normalmente rilevabili nel latte vaccino (3.2-3.5). Differenze con questo latte sono riscontrabili anche nella composizione quali-quantitativa della frazione azotata. II contenuto in NPN rispetto all'azoto totale risulta più elevato ( $15 \%$ vs valori max del $7 \%$ del latte vaccino) probabilmente a causa delle intense attività enzimatiche endogene del latte d'asina, e contribuisce al buon profilo nutrizionale e biologico di questo latte. II contenuto in caseine $(0.71 \pm 0.09 \mathrm{~g} / 100 \mathrm{~g})$ e sieroproteine $(0.75 \pm 0.07 \mathrm{~g} / 100 \mathrm{~g})$ risulta più equilibrato (rapporto $\mathrm{CAS} / \mathrm{SP}=$ $0.95 \pm 0.16$ ) rispetto ad un valore di circa 4 nel latte vaccino.

L'analisi mediante CE-SDS, che separa le proteine in funzione del loro peso molecolare, ha consentito la risoluzione dei picchi delle diverse sieroproteine rispetto al gruppo delle caseine (Fig. 1) ed è stato così possibile calcolarne la percentuale relativa rispetto al totale delle proteine integrate: $\alpha$-lattoalbumina (14.62 $\pm 1.24, \mathrm{CV} \%$ 8.47), lisozima (9.65 \pm 0.62, CV\% 6.41), $\beta$-lattoglobulina (24.00 \pm 1.02, CV\% 4.24), sieroalbumina $(1.20 \pm 0.22$, CV\% 18.00$)$ e caseine $(50.10 \pm 2.44, \mathrm{CV} \%$ 4.86). Complessivamente, il rapporto caseine/sieroproteine calcolato con questa tecnica e pari a $1.01 \pm 0.10$, risulta in buon accordo con il valore calcolato dall'analisi chimica delle proteine e conferma I'accuratezza della metodica elettroforetica applicata. L'analisi CE-SDS ha messo in luce l'elevato contenuto di lisozima del latte d'asina, la cui corretta quantificazione ha previsto l'applicazione della tecnica CZEUREA. La consistente presenza di questo enzima nel latte d'asina ha stimolato studi sul suo possibile utilizzo, nella produzione di formaggi duri a lunga maturazione, per contrastare lo sviluppo dei clostridi, responsabili del difetto di gonfiore (20).

La frazione minerale (Tab. I), espressa come contenuto in ceneri, risulta inferiore rispetto al latte vaccino $(0.7-0.8 \mathrm{~g} / 100 \mathrm{~g})$, mentre il contenuto in lattosio è decisamente più elevato e responsabile della maggiore appetibilità. Oltre al lattosio, in questo lavoro, è stata individuata anche la presenza, se pur in concentrazioni minime, di glucosio e galattosio (9 e $14 \mathrm{mg} / 100 \mathrm{~g}$, rispettivamente). Tali valori sono confrontabili con quelli del latte vaccino (circa $10 \mathrm{mg} / 100 \mathrm{~g}$ per entrambi gli zuccheri (21)). Della medesima entità è risultata anche la concentrazione in acido lattico (circa $12 \mathrm{mg} / 100 \mathrm{~g}$ ), mentre l'acido citrico pur essendo 10 volte più concentrato, è inferiore ai valori del latte vaccino (153-260 mg/100g (22)). A nostra conoscenza non sono presenti dati di letteratura relativi al latte d'asina, né su glucosio e galattosio, né sui due acidi organici citati.

La Figura 2 mostra i profili dei trigliceridi del grasso di latte di asina e

\section{Tabella I - Caratteristiche compositive del latte di asina}

Table I - Compositional characteristics of donkey milk

\begin{tabular}{|c|c|c|c|c|}
\hline & media & dev.std & Dati bibliografici & Ref \\
\hline $\mathrm{pH}$ & 7.07 & 0.12 & 7.0-7.35; 6.7-7.6 & $2 ; 19$ \\
\hline Grasso (g/100g) & 0.46 & 0.23 & $0.3-1.8 ; 0.02-2.05$ & $2 ; 19$ \\
\hline Proteine $(\mathrm{g} / 100 \mathrm{~g})$ & 1.71 & 0.12 & $1.3-1.9 ; 0.21-2.88$ & $2 ; 19$ \\
\hline Caseina $(g / 100 g)$ & 0.71 & 0.09 & $0.64-1.03$ & 25 \\
\hline Sieroproteine $(\mathrm{g} / 100 \mathrm{~g})$ & 0.75 & 0.07 & $0.49-0.80$ & 25 \\
\hline NPN (g/100g) & 0.04 & 0 & $0.03-0.04$ & 2 \\
\hline NPN/Ntot x 100 & 15.03 & & $14-16$ & 2 \\
\hline Caseina/Sieroproteine & 0.95 & 0.16 & $0.8-1.2$ & 2 \\
\hline Caseina \% * & 50.1 & 2.44 & $37-40$ & 25 \\
\hline a-lattoalbumina \% * & 14.62 & 1.24 & $12-14$ & 25 \\
\hline Lisozima \% * & 9.65 & 0.62 & $13-15$ & 25 \\
\hline$\beta$-lattoglobulina \% * & 24 & 1.02 & $21-23$ & 25 \\
\hline Sieroalbumina \% * & 1.2 & 0.22 & $2-3$ & 25 \\
\hline Lisozima $(\mathrm{g} / 100 \mathrm{ml})^{\star \star}$ & 0.14 & 0.01 & 0.1 & 20 \\
\hline Ceneri (g/100g) & 0.38 & 0.04 & $0.3-0.5$ & 2 \\
\hline Lattosio (g/100g) & 6.82 & 0.14 & $5.8-7.4 ; 3.5-8.5$ & $2 ; 19$ \\
\hline Glucosio(mg/100g) & 9.24 & 0.96 & & \\
\hline Galattosio $(\mathrm{mg} / 100 \mathrm{~g})$ & 13.97 & 2.86 & & \\
\hline Ac. citrico $(\mathrm{mg} / 100 \mathrm{~g})$ & 135.19 & 17.31 & & \\
\hline Ac. lattico $(\mathrm{mg} / 100 \mathrm{~g})$ & 12.14 & 3.03 & & \\
\hline
\end{tabular}

*Valori espressi in percento sulle proteine ottenute mediante CE-SDS

* Values expressed as percentage of total proteins obtained by CE-SDS

** Valori ottenuti con la tecnica CZE-UREA

** Values obtained by CZE-UREA

di vacca. La tecnica gascromatografica adottata consente la separazione in base al numero di atomi di carbonio dei 3 acidi grassi che costituiscono i trigliceridi, indipendentemente dal grado di insaturazione o da eventuali isomerie. Di conseguenza, data la grande varietà di acidi grassi che costituiscono il grasso di latte, in ogni picco coeluiscono diversi trigliceridi costituiti da acidi grassi differenti, con uguale somma del numero di atomi di carbonio. Nel latte vaccino i trigliceridi mostrano una caratteristica distribuzione bimodale con due massimi a 38 e 50 atomi di carbonio. Nel grasso del latte d'asina, la distribuzione mantiene una forma bimodale, ma i due massimi si posizionano sui trigliceridi a 44 e 52 atomi di carbonio, contenenti acidi grassi a più lunga catena. Inoltre, il grasso del latte d'asina mostra, mediamente una maggiore concentrazione di trigliceridi a più alto numero di atomi di carbonio, rispetto al latte vaccino. Questo risultato è dovuto alla scarsa presenza, nel latte d'asina, di trigliceridi contenenti acidi grassi saturi a catena corta, caratteristici del grasso del latte proveniente da animali ruminanti.

La determinazione dei singoli acidi grassi conferma quanto la distribuzione dei trigliceridi lasciava supporre e mostra altre interessanti 


\section{Tabella II - Composizione delle maggiori classi di acidi grassi del latte d'asina}

Table II - Composition of the main classes of fatty acids of donkey milk

\begin{tabular}{ccccc} 
Categorie di acidi grassi & media & dev.std & Dati bibliografici & Ref \\
\hline Saturi (SFA) & 58.2 & 3.6 & $47-67$ & 1 \\
\hline Insaturi (UFA) & 41.9 & 3.7 & $33-53$ & 1 \\
\hline Monoinsaturi (MUFA) & 24.7 & 2.9 & $15-35$ & 1 \\
\hline Polinsaturi (PUFA) & 17.2 & 2.3 & $15-30$ & 1 \\
\hline
\end{tabular}

differenze con il latte vaccino (Fig. 3a). Il grasso del latte d'asina presenta concentrazioni molto ridotte di acido butirrico ( $C 4=0.3 \% \pm$ $0.08)$ e di acido caproico $(\mathrm{C} 6=0.55 \pm 0.1)$ mentre C8 $(4.5 \% \pm 0.9)$, C10 $(9.5 \% \pm 1.9)$ e $\mathrm{C} 12(9.3 \% \pm 1.4)$ risultano più concentrati dei valori medi del grasso di latte vaccino. Concentrazioni maggiormente confrontabili con il latte vaccino si ritrovano per l'acido miristico (C14\% $=8.6 \pm 1.1)$ e palmitico $(C 16 \%=22.0 \pm 1.7)$. Particolarmente interessante è la composizione degli acidi a 18 acidi di carbonio. Oltre alla bassa concentrazione del C18 saturo (acido stearico $=1.6 \% \pm 0.3$ ) è da sottolineare la quasi totale assenza di acidi nella configurazione trans (Fig. 3b), che sono invece caratteristici del grasso di latte vaccino. Essi infatti derivano dalle reazioni di bioidrogenazione e successiva desaturazione che avvengono nel rumine ad opera di specifici microrganismi. Di conseguenza, il grasso di latte di asina è anche privo di acido linoleico coniugato (CLA), mentre è maggiormente ricco in acido linolenico $(C 18: 3=10.8 \% \pm 1.7)$ e linoleico $(C 18: 2=5.9 \% \pm 1.1)$. La Tabella II riporta la composizione in acidi grassi raggruppati nelle principali classi, sulla base della presenza/assenza dei doppi legami. I risultati sono in linea con i dati bibliografici la cui variabilità è probabilmente dovuta alla maggiore e diretta correlazione tra composizione lipidica della dieta e composizione del grasso di latte che si verifica negli animali monogastrici.

Dal punto di vista nutrizionale non vi è dubbio che il grasso del latte d'asina abbia una maggiore valenza, secondo le linee guida per una sana alimentazione (Fig. 4). Oltre all'elevato contenuto di acidi polinsaturi sia della serie omega-6, che omega-3, è interessante notare come sia favorevole il rapporto tra questi due gruppi di costituenti e maggiormente in linea con i valori consigliati, che variano da 1 a 4 secondo quanto riportato da Simopoulos (23). Anche il rapporto tra acidi saturi ed insaturi è inferiore rispetto al latte vaccino e, come è stato già osservato non vi è presenza significativa di acidi trans. A questo proposito è però opportuno sottolineare che gli acidi grassi trans, naturalmente presenti nel grasso del latte dei ruminanti, non hanno $\mathrm{i}$ medesimi effetti negativi sulla salute degli acidi trans derivanti dalla

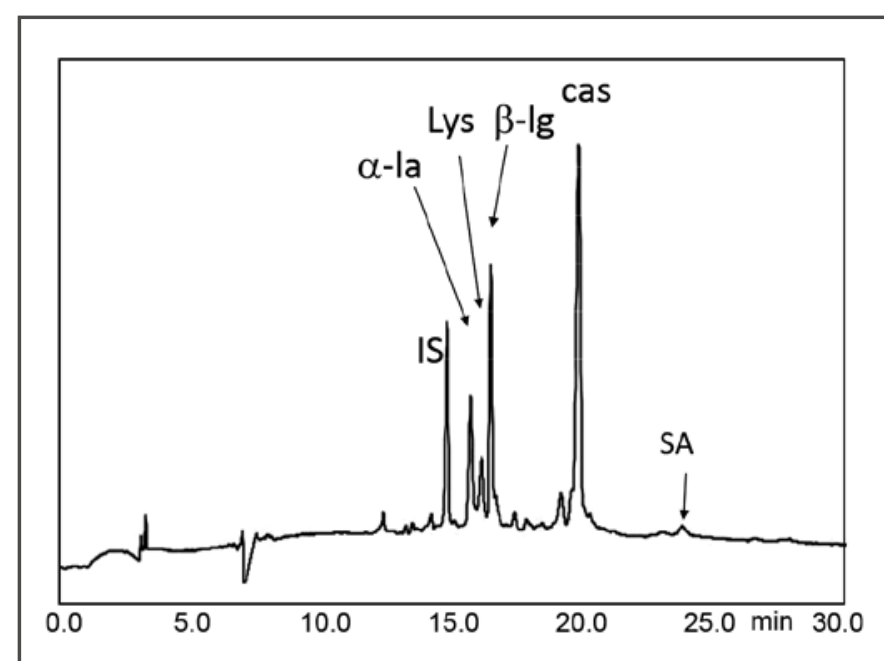

Figura 1 - Profilo elettroforetico CE-SDS delle proteine del latte d'asina: IS=standard interno, proteina da $10 \mathrm{kDa}$; $\alpha$-la=a-lattoalbumina; Lys=lisozima; $\beta$-lg= $\beta$-lattoglobulina; cas=caseina; $\mathrm{SA}=$ sieroalbumina.

Figure 1 - CE-SDS electrophoretic profile of donkey milk proteins: $I S=$ internal standard, $10 \mathrm{kDa}$ protein; $\alpha-l a=\alpha-l a c t a l b u m i n ; ~ L y s=l y s o z y m e ; ~ \beta-l g=\beta-$ lactoglobulin;cas=casein; $S A=$ serum albumin .

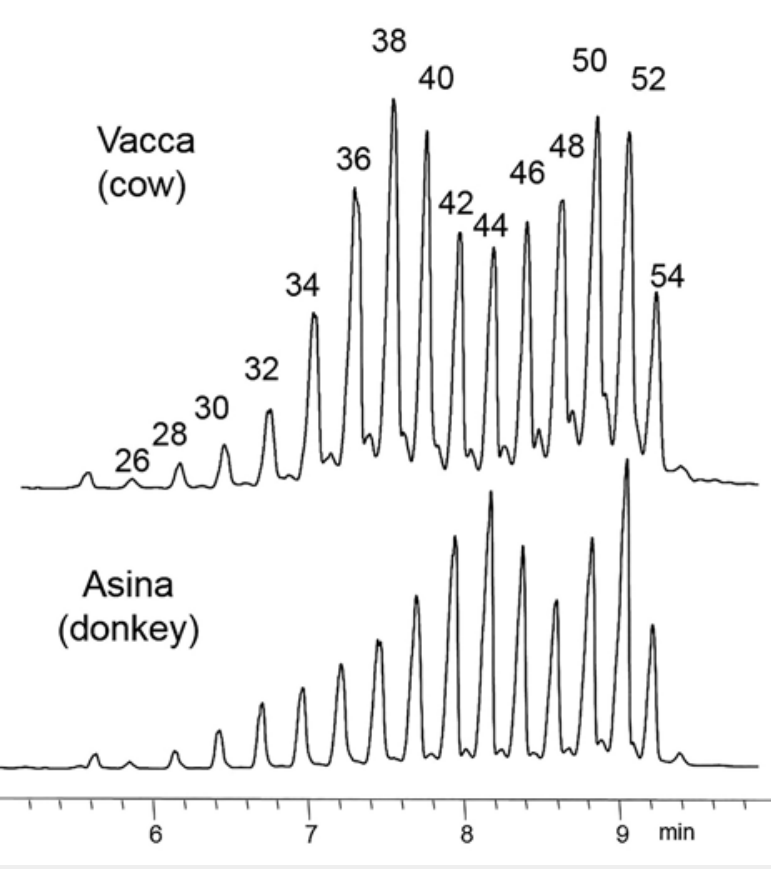

Figura 2 - Profilo gascromatografico dei trigliceridi del latte vaccino e del latte di asina.

Figure 2 - Gaschromatographic profile of triglycerides of cow and donkey milk 

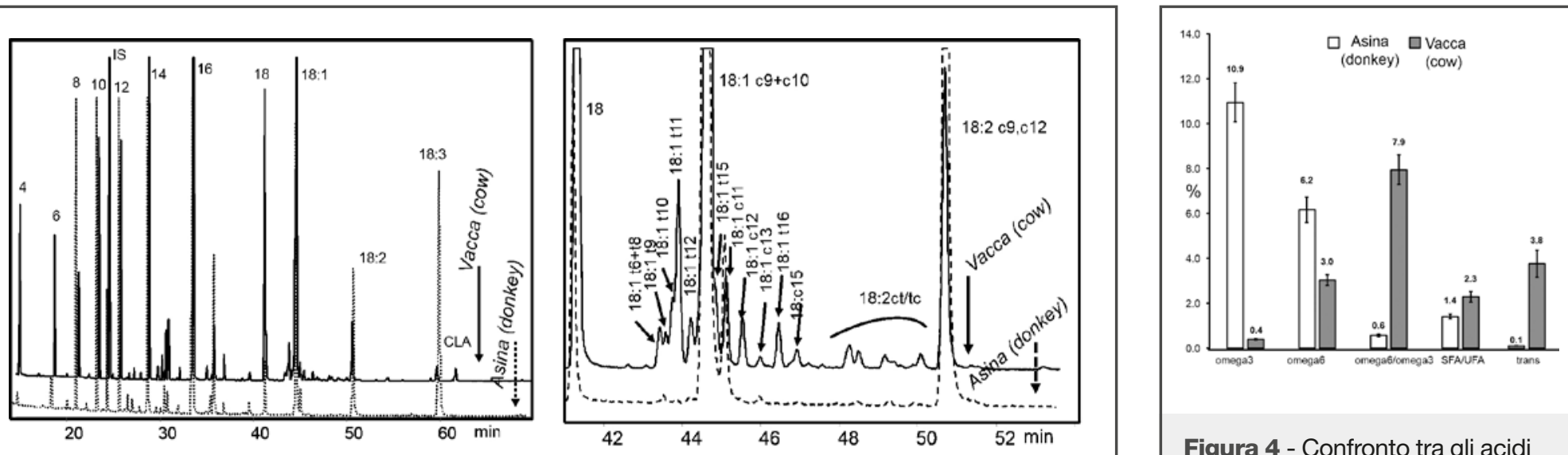

Figura 3 - Profilo gascromatografico degli acidi grassi del latte vaccino e del latte d'asina e visione allargata della zona di eluizione degli acidi a 18 atomi di carbonio

Figure 3 - Gaschromatographic profile of fatty acids of cow and donkey milk and enlarged view of the elution area of fatty acid with 18 carbon atoms

lavorazione industriale e presenti in oli parzialmente idrogenati (24). Ciò è probabilmente dovuto alla diversità, esistente tra le due tipologie di grassi, nella posizione dell'isomeria del doppio legame sulla catena carboniosa. Nel grasso di latte dei ruminanti infatti l'isomero trans maggiormente rappresentato è l'acido vaccenico (C18:1 trans-11), mentre nei prodotti parzialmente idrogenati è l'acido elaidinico (C18:1 trans-9).

II contenuto in colesterolo del grasso di latte d'asina (388 \pm 48 $\mathrm{mg} / 100 \mathrm{~g}$ di grasso) è circa il $40 \%$ più elevato di quello del grasso di latte vaccino. Tuttavia, data l'esigua quantità di grasso mediamente presente in questa tipologia di latte, ciò non rappresenta un problema dal punto di vista nutrizionale (Tab. III).

La composizione in fosfolipidi, in termini percentuali è in linea con $i$ dati bibliografici relativi al latte d'asina e simile a quella del latte vaccino, ad eccezione del contenuto in sfingomielina che risulta inferiore. Leggermente più elevato appare invece il contenuto totale che si posiziona su valori pari all' $1 \%$ del grasso totale, mentre nel latte vaccino i valori sono mediamente più bassi. II ridotto contenuto di grasso totale del latte d'asina determina, però, una scarsa concentrazione di questi costituenti nel latte stesso.

\section{CONCLUSIONI}

La ricerca svolta ha permesso di ampliare la conoscenza sul latte d'asina e rappresenta un contributo alla definizione delle sue caratteristiche, sia per il settore zootecnico e lattiero-caseario, sia per la comunità scientifico-medica. I risultati ottenuti hanno confermato che la particolare composizione giustifica le caratteristiche di digeribilità e tollerabilità, che rendono il latte d'asina un prodotto funzionale. La composizione proteica ha confermato le

\section{Tabella III - Composizione fosfolipidica del latte d'asina espressa in percentuale sul totale dei fosfolipidi}

Table III - Phospholipid composition of donkey milk expressed as percentage on the total phospholipids

\begin{tabular}{ccccc}
$\%$ & media & dev.std & $\begin{array}{c}\text { Dati bibliografici } \\
\text { asina (26) }\end{array}$ & $\begin{array}{c}\text { Dati bibliografici } \\
\text { vacca (27) }\end{array}$ \\
\hline PI & 13.4 & 1.5 & $10-15$ & $3-14$ \\
\hline PE & 35.2 & 3.0 & $31-40$ & $29-46$ \\
\hline PS & 6.0 & 0.7 & $9-11$ & $2-16$ \\
\hline PC & 24.7 & 2.6 & $17-26$ & $21-45$ \\
\hline SM & 20.8 & 1.4 & $14-18$ & $29-46$ \\
\hline $\begin{array}{c}\text { PL totali } \\
(\mathrm{mg} / \text { 100g grasso) }\end{array}$ & 1032.6 & 161.6 & $650-1230$ & $400-1000$ \\
\hline $\begin{array}{c}\text { PL totali } \\
(\mathrm{mg} / \text { 100g latte) }\end{array}$ & 4.6 & 2.1 & $2.7-6.1$ & $14-34^{*}$
\end{tabular}

* Valori calcolati sulla base di un contenuto medio del grasso di latte vaccino pari a 3.4\% * Values calculated on the basis of a cow milk fat mean content of $3.4 \%$

$(\mathrm{PI}=$ fosfatidil inositolo, $\mathrm{PE}=$ fosfatidil etanolammina, $\mathrm{PS}=$ fosfatidil serina, $\mathrm{PC}=$ fosfatidi colina, $\mathrm{SM}=$ sfingomielina, $\mathrm{PL}=$ fosfolipidi).

(PI=phosphatidylinositol, $\mathrm{PE}=$ phosphatidylethanolamine, $\mathrm{PS}=$ phosphatidylserine, $\mathrm{PC}=$ phosphatidylcholine, $\mathrm{SM}=$ sphingomyelin, $\mathrm{PL}=$ phospholipid).

informazioni quali-quantitative riportate in bibliografia. Inoltre, è bene sottolineare che, sebbene il latte d'asina contenga composti azotati riconducibili alle stesse classi dei principali allergeni del latte vaccino (ad es. $\beta$-lattoglobulina e alcune frazioni caseiniche), studi recenti hanno evidenziato una bassa omologia di sequenza tra le proteine del latte delle due specie. Le differenze tra le sequenze amminoacidiche delle proteine bovine e d'asina 
determinano delle modificazioni, se non la totale assenza, dei siti di reattività con le immunoglobuline $E$ responsabili della reazione allergica e quindi una migliore tollerabilità del latte d'asina da parte dei soggetti allergici alle proteine del latte bovino.

Anche il contributo della frazione lipidica, seppur presente in basse percentuali, si conferma molto importante nel determinare il valore nutrizionale del latte d'asina, soprattutto per l'elevato contenuto in acidi grassi insaturi omega-3. Per ciò che riguarda l'elevata concen- trazione del colesterolo nella matrice lipidica, è opportuno ricordare che tale molecola, oltre ad essere un fondamentale componente delle membrane cellulari, è il precursore degli ormoni steroidei e della vitamina $\mathrm{D}$, sostanze indispensabili, soprattutto nei bambini, per una crescita equilibrata e un corretto sviluppo neurologico.

Si auspica che i dati ottenuti possano contribuire a fare chiarezza fra le numerose informazioni che si trovano sul web, spesso di incerta provenienza, in merito al latte d'asina.

\section{CONFLITTO DI INTERESSE}

Gli autori dichiarano che non esistono conflitti di interesse economico da parte di uno o più autori.

\section{BIBLIOGRAFIA}

1. Salimei E., Fantuz F. Equid milk for human consumption. Int Dairy J 2012; 24:130-142

2. Salimei E. Animals that produce dairy foods e donkey. In: Encyclopedia of dairy sciences, vol. 1 (eds. J. W. Fuquay, P. F. Fox, \& P. L. H. McSweeney), 2011, 365-373.

3. Altomonte I., Salari F., Licitra R., Martini M. Donkey and human milk: Insights into their compositional similarities. Int. Dairy J. 2019; 89:111-118.

4. Uniacke T., Fox P.F. Equid milk: Chemistry, Biochemistry and Processing. In: Food Biochemistry and Food Processing (eds John Wiley \& Sons), 2012, 491-530. 5. Cunsolo V., Saletti R., Muccilli V., Gallina S., Di Francesco A., Foti S. Proteins and bioactive peptides from donkey milk: The molecular basis for its reduced allergenic properties. Food Res. Int. 2017; 99:41-57.

6. Carminati D., Tidona F. Nutritional value and potential health benefits of donkey Milk. In: Nutrients in dairy and their Implications for health and disease, (eds. Ross Watson D, Collier R,J, Preedy V,R.), 2017, 407-414.

7. ISO Milk, cream and evaporated milk - Determination of total solids content (Reference method). ISO Standard 6731 - 2010. Geneva, Switzerland: International Organization of Standardization.

8. AOAC Ash of milk. Official method n.930.30 - 1997 In W. Horowitz (Ed.), Official methods of analysis of AOAC International. Gaithersburg, MD, USA: AOAC International.

9. ISO Milk and milk products - Determination of nitrogen content - Part 1: Kjeldahl principle and crude protein calculation ISO Standard 8968-1 - 2014. Geneva, Switzerland: International Organization of Standardization.

10. ISO Milk - Determination of casein-nitrogen content - Part 1: Indirect method (Reference method) ISO Standard 17997-1 -2004 - Geneva, Switzerland: International Organization of Standardization.

11. ISO Milk - Determination of fat content. ISO Standard 2446 - 2008. Geneva, Switzerland: International Organization of Standardization.

12. Bouzas J., Kantt C.A., Bodyfelt F., Torres A. Simultaneous determination of sugars and organic acids in cheddar cheese by high-performance liquid chromatography. J Food Sci 1991; 56: 276-278.

13. Recio I., Olieman C. Determination of denatured serum proteins in the casein fraction of heat treated milk by capillary zone electrophoresis. Electrophoresis 1996; 17:1228-1233.

14. Folch L., Less M., Stanley G.H.S. A simple method for the isolation and purification of total lipids from animal tissues. J Biol Chem 1957; 726:497-509.
15. 15) ISO Milk and milk products - Determination of milk fat purity by gas chromatographic analysis of triglycerides (Reference method) ISO Standard 17678 - 2019. Geneva, Switzerland: International Organization of Standardization.

16. Contarini G., Povolo M., Pelizzola V., Monti L., Lercker G. Interlaboratory evaluation of milk fatty acid composition by using different $\mathrm{GC}$ operative conditions. J Food Comp An 2013; 32:131-140.

17. Avalli A., Contarini G. Determination of phospholipids in dairy products by SPE/HPLC/ELSD. J Chromatogr A 2005; 170:107-190.

18. ISO Anhydrous milk fat- Determination of sterol composition by gas liquid chromatography (Routine method) ISO Standard 18252 - 2006. Geneva, Switzerland: International Organization of Standardization.

19. Malissiova E., Arsenos G., Papademas P., Fletouris D., Manouras A., Aspri $M$, et al. Assessment of donkey milk chemical, microbiological and sensory attributes in Greece and Cyprus. Int J Dairy Technol 2016; 69: 143-146.

20. Niro S., Fratianni A., Colavita G., Galassi L., Zanazzi M., Salimei E. Technological use of donkey milk in cheesemaking. Int J Dairy Technol 2017; 70: 439-442. 21. Ohlsson J.,A., Johansson M., Hansson H., Abrahamson A., Byberg L., Smedman A., et al. Lactose, glucose and galactose content in milk, fermented milk and lactose-free milk products. Int Dairy J 2017; 73:151-154.

22. Ducháček J., Stádník L., Beran J., Okrouhlá M. The relationship between fatty acid and citric acid concentrations in milk from Holstein cows during the period of negative energy balance. JCEA 2012; 13:615-630.

23. Simopoulos A.,P. The importance of the ratio of omega-6/omega-3 essential fatty acids. Biomed Pharmacother 2002; 56:365-379.

24. Wang Y., Jacome-Sosa M.,M., Proctor S.,D. The role of ruminant trans fat as a potential nutraceutical in the prevention of cardiovascular disease. Food Res Inter 2012; 46:460-468.

25. Guo H., Y, Pang K., Zhang X., Y., Zhao L., Chen S., W., Dong M., L., et al. Composition, physiochemical properties, nitrogen fraction distribution, and amino acid profile of donkey milk. J Dairy Sci 2007; 90:1635-1643.

26. Contarini G., Pelizzola V., Scurati S., Povolo M. Polar lipid of donkey milk fat: phospholipid, ceramide and cholesterol composition. J Food Comp An 2017; 57:16-23.

27. Contarini G., Povolo M. Phospholipids in milk fat: composition, biological and technological significance, and analytical strategies. Int J Mol Sci 2013; 14:2808-2831. 